\title{
Young stellar populations in early-type galaxies in the SDSS
}

\author{
Louisa A. Nolan ${ }^{1}$, Ata Kabán ${ }^{2}$, Markus Harva ${ }^{3}$, Andrew Benson ${ }^{4}$ \\ and Somak Raychaudhury ${ }^{1}$ \\ ${ }^{1}$ School of Physics and Astronomy, University of Birmingham, Birmingham, B15 2TT, UK \\ email: lan@star.sr.bham.ac.uk \\ ${ }^{2}$ School of Computer Science, University of Birmingham, Birmingham, B15 2TT, UK \\ ${ }^{3}$ Laboratory of Computer and Information Science, Helsinki University of Technology, \\ Helsinki, PO Box 5400, FI-02015 HUT, Finland \\ ${ }^{4}$ California Institute of Tech., MC 130-33, 1200 E. California Blvd., Pasadena, CA 91125, USA
}

\begin{abstract}
We use a purely data-driven rectified factor analysis to identify early-type galaxies with young ( $\approx 4$ Gyr) stellar populations in the Sloan Digital Sky Survey Spectroscopic Catalogue. We call these galaxies $\mathrm{E}+\mathrm{F}$ galaxies, analogous to $\mathrm{E}+\mathrm{A}$ galaxies. These galaxies lie in the 'Green Valley', between the blue cloud and the red sequence on the colour-magnitude diagram. As such, these galaxies may represent an important transient stage in the evolution of galaxies from blue and star-forming to red and passive. We investigate the distribution in projected local galaxy surface density of the $\mathrm{E}+\mathrm{F}$ galaxies, and compare it with the environment of earlytype and $\mathrm{E}+\mathrm{A}$ galaxies. We find that $\mathrm{i}$ ) the $\mathrm{E}+\mathrm{A}$ distribution peaks strongly in projected local galaxy surface density, $\Sigma_{5}$, at $\sim 0.1-0.2 \mathrm{Mpc}^{-2}$, ii) early-types have a flatter peak at $\sim 0.06-$ $0.2 \mathrm{Mpc}^{-2}$, iii) the E+Fs lie somewhere in between, and iv) the distributions of the models do not agree well with the data, peaking at higher densities, and under-predicting the number of $\mathrm{E}+\mathrm{As}$ at low $\left(\Sigma_{5}<0.3 \mathrm{Mpc}^{-2}\right)$ densities. The dearth of $\mathrm{E}+\mathrm{A}$ and $\mathrm{E}+\mathrm{F}$ galaxies in dense environments confirms that $\mathrm{E}+\mathrm{A}$ and $\mathrm{E}+\mathrm{F}$ galaxies are most likely the products of galaxy-galaxy merging or interactions, rather than star-forming galaxies whose star formation has been quenched by processes unique to dense environments, such as ram-pressure stripping or galaxy harassment. The similarity of the environments in which the $\mathrm{E}+\mathrm{F}$ population and the $\mathrm{E}+\mathrm{A}$ galaxy sample are found, together with the spectral evidence, suggests that $\mathrm{E}+\mathrm{F}$ galaxies are $\mathrm{E}+\mathrm{A}$ galaxies, which have evolved by a further $\sim$ one to a few Gyr.
\end{abstract}

Keywords. techniques: spectroscopic; galaxies: elliptical and lenticular, $\mathrm{cD}$; galaxies: evolution; galaxies: formation; galaxies: stellar content

\section{Introduction}

Mass assembly and star formation do not appear to have a straightforward relationship. Smaller present-day early-type galaxies (ETGs) have, on average, younger stellar populations than more massive ETGs (e.g. Cowie et al. 1996, Treu et al. 2005). This seems to be in contradiction of the hugely successful hierarchical model of structure formation, where smaller objects form at earlier epochs. To understand this dichotomy, we need to know the star-formation history of ETGs, so that we may probe the relationship between mass assembly, environment, and the triggering and quenching of star formation.

\section{2. $\mathbf{E}+\mathbf{F}$ galaxies in the SDSS}

We select a sample of $>10,000$ ETGs from the SDSS DR4 spectroscopic catalogue. Following Bernardi et al. (2003), we select on concentration index (r90/r50 > 2.5 in i*), and surface brightness profile. We also have a sample of $96 \mathrm{E}+\mathrm{A}$ galaxies, selected for the absence of emission lines and the presence of strong Balmer absorption lines. In both 


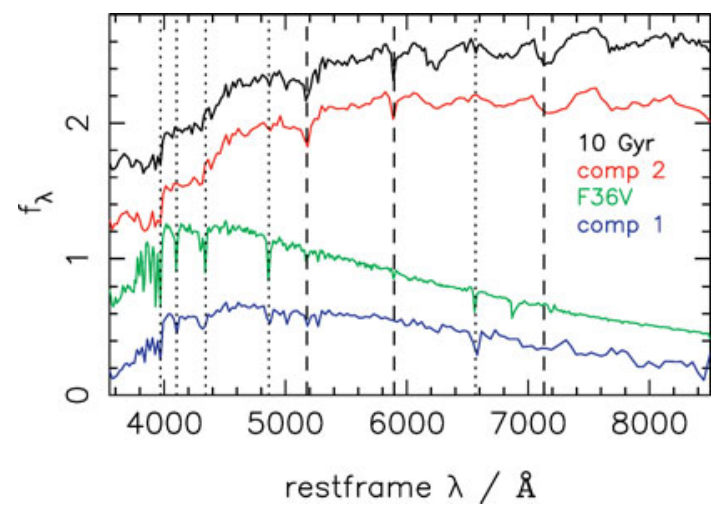

Figure 1. Components recovered from our factor analysis of SDSS ETG spectra. From top to bottom: a $10 \mathrm{Gyr}, 2.5 \mathrm{Z} \odot$ single stellar population model (Bruzual \& Charlot 2003); component 2 from our analysis; a super-solar metallicity F star spectrum (Santos et al. 1995); component 1 from our analysis. The dotted lines mark some of the absorption features in the spectra which are typically strong in young stellar populations, and the dashed lines mark some of the absorption features which are typically strong in old, metal-rich stellar populations. From left to right, the absorption line species are: $\mathrm{H} \varepsilon, \mathrm{H} \delta, \mathrm{H} \gamma, \mathrm{H} \beta, \mathrm{Mgb}, \mathrm{NaD}, \mathrm{H} \alpha$, TiO. It can clearly be seen that the first component represents a younger stellar contribution, whereas the second component represents a mature stellar population.

cases, the redshift range is restricted to $0.06<\mathrm{z}<0.122$, and galaxies with absolute R-band magnitudes, $M_{R}<-21$, for which the SDSS is complete at these redshifts. Full details of the selection can be found in Nolan, Raychaudhury \& Kabán (2007), and Nolan, Benson \& Raychaudhury (2007, in preparation).

We use a purely data-driven rectified factor analysis (RFA) developed by Harva \& Kabán (2005) to identify ETGs in our sample which contain young stellar populations. Our hypothesis is that each of the $N$ observations of galaxy spectra (here, $N>10,000$ ) can be described by a superposition of $K$ underlying (and as yet unknown) components, where $K<N$. The $K$ components are assumed to be statistically independent, and we include terms for both the noise of the model, and also for the observational errors. In our RFA model, both the underlying components and the weights of these components in the linear superposition have a positivity restriction. This gives us a more physically realistic flux model than if we allowed negative values. In Nolan et al. (2007), we find that the spectra of ETGs is well-described by two underlying components, so we set $K=$ 2. For a complete description of the rectified factor analysis, see Nolan et al. (2007).

Figure 1 shows the two components recovered from the analysis of the SDSS ETG spectra. One component represents an old, metal-rich population, and the other a young stellar population. The 'young' component looks like the spectrum of an F-star, at $\sim 4$ Gyr, also shown in Figure 1. Hence, we can construct a population of ETGs which contain a significant $(\gtrsim 10 \%)$ young $(\lesssim 4 \mathrm{Gyr})$ stellar component, by choosing those ETGs for which the weight of the first, 'young' component is greater than that of the second component. We call these 'E+F' galaxies, in analogy to E+As. A detailed discussion of the components and identification of $\mathrm{E}+\mathrm{F}$ galaxies can be found in Nolan, Raychaudhury \& Kabán (2007).

Using the above criterion, we identify $970 \mathrm{E}+\mathrm{F}$ galaxies in our sample of $>10,000$ ETG spectra. Figure 2 shows the $(g-r)-M_{R}$ diagram for SDSS galaxies in the redshift range $0.06<\mathrm{z}<0.08$. The $\mathrm{E}+\mathrm{F}$ galaxies lie predominantly in the so-called 'Green valley', between the blue cloud and the red sequence. This suggests that these relatively uncommon objects represent a transition stage between the blue, star-forming population and passive red galaxies. We next explore the environments of these galaxies, to investigate the mechanisms for this transformation. 


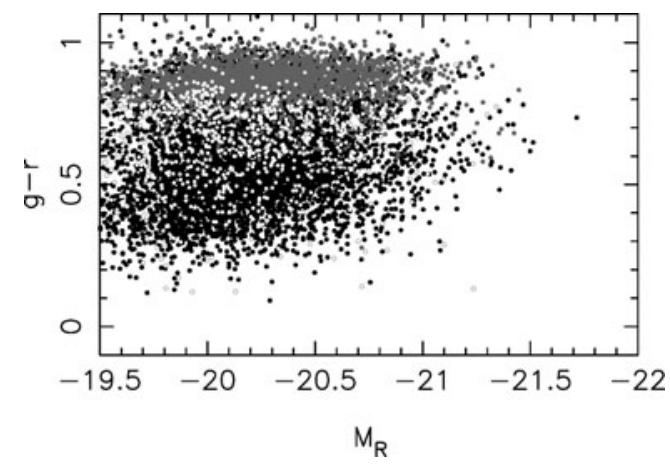

Figure 2. Colour-magnitude diagram for SDSS galaxies showing our $\mathrm{E}+\mathrm{F}$ galaxies (white circles) situated in the 'Green Valley' between the red sequence (grey) and the blue cloud (black).

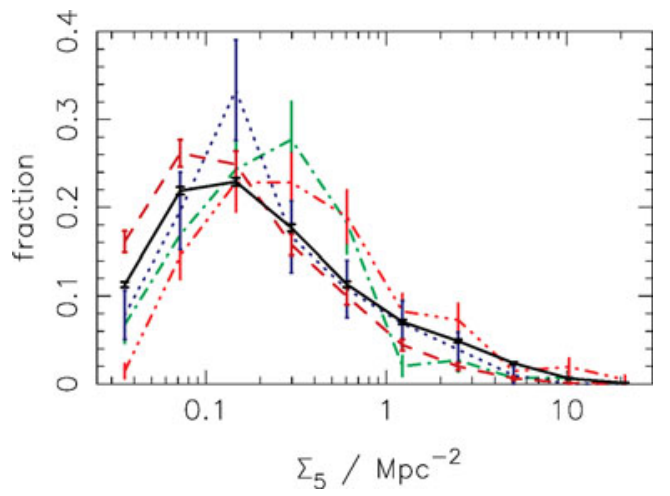

Figure 3. Normalised distributions of projected local surface density, $\Sigma_{5}$ of: ETGs (solid line); $\mathrm{E}+\mathrm{Fs}$ (dashed line); E+As (dotted line) from Sloan; E+As from Baugh et al. (2005) (dashdot-dash-dot) E+As from Bower et al. (2006) (dash-dot-dot-dot). 'E+As' in the models are defined as those ETGs which have formed at least $10 \%$ of their stars in a merger in the last 1 Gyr. The populations are normalised so that the area under each line is unity.

\section{Environment}

We can use our large sample of $\mathrm{E}+\mathrm{F}$ galaxies to explore the physical mechanisms which trigger / quench starbursts during the formation of ETGs. Figure 3 shows the distribution of the projected local galaxy surface density, $\Sigma_{5}$, for each of our SDSS galaxy population: ETGs, E+Fs and E+As, compared with the distribution of ETGs which have formed at least $10 \%$ (by mass) of their stars in a merger-induced starburst in the last 1 Gyr from the semi-analytic simulations of Baugh et al. (2005) and Bower et al. (2006) at $z=0.089$. There are four things to note from the plot: i) the distribution of the E+As peaks strongly at $\Sigma_{5} \sim 0.1-0.2 \mathrm{Mpc}^{-2}$; ii) the distribution of the ETGs peaks less strongly, at a similar density $\Sigma_{5} \sim 0.05-0.2 \mathrm{Mpc}^{-2}$; iii) the distribution of the $\mathrm{E}+\mathrm{Fs}$ lies somewhere in between these two; iv) the model $\mathrm{E}+\mathrm{A}$ distributions do not reproduce the data well. They underpredict the number of E+As at low densities $\left(\Sigma_{5} \lesssim 0.3 \mathrm{Mpc}^{-2}\right)$, and overpredict their number at intermediate (Baugh et al. 2005; Bower et al. 2006) and high (Bower et al. 2006) densities.

The peak in the $\mathrm{E}+\mathrm{A}$ distribution at $\sim 0.1-0.2 \mathrm{Mpc}^{-2}$ may indicate the optimum environment for galaxy-galaxy mergers and interactions, representing a trade-off between sufficient density for galaxy-galaxy encounters, and slow enough relative velocities that these encounters lead to a meaningful interaction. One would then expect that, if these 
galaxy-galaxy encounters represent the main channel for creating ETGs, we would see the majority of ETGs residing in environments which favour these meaningful interactions. Further, we might expect the peak to be somewhat more spread-out as galaxy group dynamics evolve. This is in fact what we see, with the $\mathrm{E}+\mathrm{F}$ galaxy distribution lying somewhere between the ETG and E+A distributions, which is strongly suggestive of $\mathrm{E}+\mathrm{Fs}$ as evolved $\mathrm{E}+\mathrm{As}$. The dearth of $\mathrm{E}+\mathrm{A}$ and $\mathrm{E}+\mathrm{F}$ galaxies in dense environments confirms that $\mathrm{E}+\mathrm{A}$ and $\mathrm{E}+\mathrm{F}$ galaxies are most likely the products of galaxy-galaxy merging or interactions, rather than star-forming galaxies whose star formation has been quenched by processes unique to dense environments, such as ram-pressure stripping or galaxy harassment. We suggest that a more realistic modelling of galaxy-galaxy mergers / interaction in low-density environments is necessary to reproduce the data at $\Sigma_{5} \lesssim 0.5$ $\mathrm{Mpc}^{-2}$ (e.g. Hopkins et al. 2007). This will be explored further in Nolan, Benson \& Raychaudhury (2007, in preparation).

\section{Conclusions}

Using our data-driven modelling technique, we have extracted a population of ETGs with young stellar populations from the SDSS DR4. The young stellar populations are dominated by F-stars, so we call this population ' $\mathrm{E}+\mathrm{F}$ ' galaxies, analogous to $\mathrm{E}+\mathrm{As}$. $\mathrm{E}+\mathrm{Fs}$ are thus a longer-lived phenomenon than $\mathrm{E}+\mathrm{A}$ galaxies, and hence represent an important population for studying ETGs with recent star-formation. From their position in the 'Green Valley' of the colour-magnitude diagram, we suggest that E+Fs represent an important transition stage between blue, star-forming galaxies and red, passive galaxies.

We find that i) the $\mathrm{E}+\mathrm{A}$ distribution peaks strongly in projected local galaxy surface density, $\Sigma_{5}$, at $\sim 0.1-0.2 \mathrm{Mpc}^{-2}$, ii) ETGs have a flatter peak at $\sim 0.06-0.2 \mathrm{Mpc}^{-2}$, iii) the $\mathrm{E}+\mathrm{Fs}$ lie somewhere in between, and iv) the distributions of the models do not agree well with the data, peaking at higher densities, and under-predicting the number of $\mathrm{E}+$ As at low $\left(\Sigma_{5}<0.3 \mathrm{Mpc}^{-2}\right)$ densities. A more realistic modelling of galaxy-galaxy merging in low-density environments is required.

The dearth of $\mathrm{E}+\mathrm{A}$ and $\mathrm{E}+\mathrm{F}$ galaxies in dense environments confirms that $\mathrm{E}+\mathrm{A}$ and $\mathrm{E}+\mathrm{F}$ galaxies are most likely the products of galaxy-galaxy merging or interactions, rather than star-forming galaxies whose star formation has been quenched by processes unique to dense environments, such as ram-pressure stripping or galaxy harassment. The peak in the density distribution of $\mathrm{E}+\mathrm{A}$ galaxies may represent the local galaxy density at which the rate of galaxy-galaxy merging or interaction peaks. The similarity of the environments in which the $\mathrm{E}+\mathrm{F}$ population and the $\mathrm{E}+\mathrm{A}$ galaxy sample are found, together with the spectral evidence, suggests that $\mathrm{E}+\mathrm{F}$ galaxies are $\mathrm{E}+\mathrm{A}$ galaxies, which have evolved by a further $\sim$ one to a few Gyr.

\section{References}

Bernardi, M., et al. 2003, AJ, 125, 1817

Baugh, C. M., Lacey, C. G., Frenk, C. S., Granato, G. L., Silva, L., Bressan, A., Benson, A. J., \& Cole, S. 2005, MNRAS, 356, 1191

Bower, R. G., Benson, A. J., Malbon, R., Helly, J. C., Frenk, C. S., Baugh, C. M., Cole, S., \& Lacey, C. G. 2006, MNRAS, 370, 645

Bruzual, G. \& Charlot, S. 2003, MNRAS, 344, 1000

Cowie L. L., Songaila A., Hu E. M., \& Cohen J. G. 1996, AJ, 112, 839C

Harva M., Kabán A., 2005, inProc. IEEE Int. Conf. Neural Networks, p. 185

Hopkins, P. F., Cox, T. J., Keres, D., \& Hernquist, L. 2007, ArXiv e-prints, 706, arXiv:0706.1246

Nolan L. A., Harva M. O., Kabán A., \& Raychaudhury S. 2006, MNRAS, 366, 321

Nolan, L. A., Raychaudhury, S., \& Kabán, A. 2007, MNRAS, 375, 381

Santos, J. F. C., Jr., Bica, E., Dottori, H., Ortolani, S., \& Barbuy, B. 1995, A\&A, 303, 753

Treu T., Ellis R. S., Liao T. X., \& van Dokkum P. G. 2005, ApJ, 622, L5 